\title{
OSTEOPOROSIS - AN UPDATE
}

\author{
Lamichhane A P \\ TU Teaching Hospital, Maharajgunj, Kathmandu, Nepal
}

\begin{abstract}
Osteoporosis is a systemic disease characterized by decrease in bone mass per unit volume, compromised bone strength, which predisposes the affected bone to fracture. This is currently one of the leading causes of morbidity and mortality among elderly over the world. In general, osteoporosis is a silent and progressive disorder that is often brought to attention of the patients or physician only after a fracture. The aetiology of osteoporosis is multifactorial and is related to two main processes: acquisition of peak bone density that occurs at the end of the third decade and loss of bone at menopause, going on to old age. The cardinal features of osteoporosis are pain, fracture and deformity. Bone mineral density measurement is the most reliable diagnostic tool in the early stage of osteoporosis. Management of osteoporosis involves prevention and treatment. The best treatment for osteoporosis is prevention. The risk of osteoporosis can be reduced by increasing peak bone mass or by decreasing the bone loss. It needs to be emphasized that bone mineral density (BMD) peaks at about age 35 and then begins to slowly decline with significant acceleration after menopause. Therefore, the most logical and cost-effective preventive strategies are to encourage young women to stop smoking and avoid excessive use of alcohol. They should also be counseled to exercise regularly and consume adequate amounts of calcium and vitamin $D$.
\end{abstract}

\section{Key Words: Osteoporosis, Fracture, Bone mineral density, Menopause.}

\section{INTRODUCTION}

Osteoporosis is the state in which bone is fully mineralized but its structure is abnormally porous and its strength is less than normal for a person of that age and sex. There is significant decrease in bone mass per unit volume of bone tissue and this is accompanied by increased fragility of the bone. Older patients are particularly prone to this entity characterized by decreased bone mass. Osteoporosis is a systemic disease characterized by compromised bone strength, which predisposes the affected bone to fracture. ${ }^{1}$ It is now recognized that both bone density and bone quality (architecture, turnover, damage/mi- cro fracture, and mineralization) are important determinants of bone strength. In general, osteoporosis is a silent and progressive disorder that is often brought to the attention of the patient or the physician only after a fracture. Osteoporosis is currently one of the leading causes of morbidity and mortality among the elderly over the world. Vertebral compression fractures are one of the earliest and most common of the osteoporotic fractures and the prevalence increases steadily with age, ranging from $20 \%$ in 50 year old postmenopausal women to $64.5 \%$ for older women. ${ }^{2}$ Other common osteoporotic fractures are hip and wrist fractures.

\footnotetext{
Address for correspondence :

Dr. Arjun Prasad Lamichhane

TU Teaching Hospital, Maharajgunj, Kathmandu, Nepal

Email: dr_ajun@yahoo.com
}

Received Date : $11^{\text {th }}$ March, 2005

Accepted Date : $23^{\text {rd }}$ May, 2005 
In 1991, experts defined osteoporosis as a systemic disease characterized by low bone mass and micro architectural deterioration of the skeleton leading to bone fragility and increased fracture risk. Histologically, it is defined as a reduction of bone (both mineral and matrix) per unit of tissue volume. As the definition did not prove practical for patient care, a World Health Organisation (WHO) panel developed the following guidelines, based on Bone Mineral Density (BMD):

Normal is defined as BMD less than or equal to 1.0 SD below the mean for peak bone mass.

Osteopenia (low bone mass) is defined as more than 1.0 but less than or equal to $2.5 \mathrm{SD}$ below the mean.

Osteoporosis is defined as more than $2.5 \mathrm{SD}$ below the mean. ${ }^{3}$

According to WHO, 13-18\% of women over 50 years have osteoporosis and $37-50 \%$ has osteopenia. The huge cost of managing this disorder should take into account treatment of this condition and management of complications, hip fracture being the most bothersome.

Fragility Fractures: Fragility fractures resulting from low trauma such as a fall from standing height affects up to one half of women and one third of men after the age of 50. These fractures are frequently associated with osteoporosis. History of a fragility fracture is among the strongest risk factors for future fracture. Therefore, optimal care of the patient with a fragility fracture includes not only treatment of the presenting fracture itself, but also evaluation and treatment of the underlying cause or causes to prevent future fractures. However, despite the availability of therapeutic agents that reduces fracture risk among osteoporotic patients who have had a fracture, most patients with fragility fractures are not evaluated for osteoporosis or treated adequately to reduce the risk of future fracture. Orthopaedic surgeons are the first and often the only physicians seen by fracture patients. Thus, they have the unique opportunity to serve as primary advocates to ensure that appropriate action is taken to reduce the risk of future fracture. ${ }^{4}$

\section{PATHOPHYSIOLOGY}

Most individuals attain their highest level of bone mass between the ages of 16-25 years. The higher this value is, the less chance there is of developing osteoporosis. The reason for this is simply that the possibility of any specific rate of bone loss (from whatever cause) leading to a critically low levels depends on the amount of bone present before bone loss begins. In men, bone is normally lost at a rate of $0.3 \%$ of total mass per year, whereas in women the rate of bone loss is as high as $0.5 \%$ per year. The accelerated rate of bone loss which occurs at a rate of $2 \%$ to $3 \%$ of total bone mass per year begins after menopause and may last from 6 to 10 years. Osteoporosis can be primary or secondary. For the purpose of description and understanding of the disease, osteoporosis (primary) has been categorized into 2 distinct syndromes. Type I known as postmenopausal osteoporosis, occurs most commonly in women within 15 to 20 years after menopause. It affects mostly trabecular bone, increasing the patient's susceptibility to vertebral compression fractures, distal radius fractures, and intertrochanteric femoral fractures. Type II osteoporosis known as senile osteoporosis, occurs in men and women over the age of 70 years with a female to male ratio of $2: 1$. It affects cortical and trabecular bone equally, predisposing patients to multiple wedge vertebral and femoral neck fractures. Thus in type I osteoporosis, estrogen deficiency plays a primary role, while in type II osteoporosis, aging and long term calcium deficiency are more important.

The aetiology of osteoporosis is multifactorial and is related to two main processes: acquisition of peak bone density that occurs at the end of the third decade and loss of bone at menopause, going on to old age. Bone remodeling is a dynamic, continuous process involving repair, maintenance and removal of old bone. Change occurs in both trabecular and cortical bones. ${ }^{5}$ Factors affecting bone remodeling includes genetics, race, gender, calcium intake, hormonal status and exercise. Black people have higher bone density than Asians or Whites. Normally, bone resorbed by osteoclast is balanced by new bone formed by osteoblasts. With aging, a protracted phase of slow bone loss occurs in both men and women, but women have accelerated bone loss at menopause, continuing for 15 to 20 years.

Thus women are not only prone to rapid deterioration of bone mass at the perimenopausal period, but also subsequently to age related osteoporosis that follows in a slower manner.

Estrogen is important to maintain bone strength. The major physiological action of estrogen is to block activation of the bone metabolic unit. This is mediated by osteoblasts which have estrogen receptors. Estrogen inhibits the synthesis of interleukins by osteoblasts; interleukins stimulate bone resorption. Estrogen regulates bone turnover and deficiency may be associated with alteration in cytokine activity. ${ }^{6}$ Estrogen inhibits bone turnover which allows the immature collagen cross-links in the newly formed collagen to stabilize the fibres by forming stable mature cross-links. ${ }^{7}$

Age related bone loss which begins in the forties, goes on through out life and affects both trabecular and cortical bone. It involves impaired calcium conservation and may be related to secondary hyperparathyroidism. Calcium absorption through 
the intestine is primarily regulated by 1,25-dihydroxyvitamin $\mathrm{D}$, the biological active form of Vitamin D. Age may also be associated with impaired osteoblastic function. ${ }^{5}$

\section{Risk Factors for Osteoporosis}

1. Genetic including family history

2. Fair skin

3. Early menopause

4. Slender built

5. Excessive alcohol consumption

6. Cigarettes smoking

7. Inactivity

8. Malnutrition

9. Inadequate calcium intake

10. Excessive phosphorous or protein intake

11. Caffeine use

12. Exercise induced amenorrhea

13. Impaired metabolism

14. Low peak bone mass in early adulthood

\section{CLINICAL FEATURES}

The cardinal features of osteoporosis are pain, fracture and deformity. In general, osteoporosis is a silent and progressive disorder that is often brought to the attention of the patient or the physician only after a fracture.

Pain: May be localized to the site of fracture and may be severe or radiate to flanks or abdomen mimicking another disease. Typically, pain occurs at the mid or lower thoracic or lumber spine. Pain from fracture usually subsides in a few weeks. Generalized bone or musculoskeletal pain suggests another disease such as osteomalacia or metastatic cancer. ${ }^{8}$

Fracture: May involve the hip, wrist, vertebrae, or other bones including ribs, pelvis or foot. Of these, the most dreaded is hip fracture because of its association with loss of independence in many and mortality of $12-24 \%$ in one year. ${ }^{6}$

Deformity: Results in change in body shape manifesting as kyphosis (Dowager's hump), loss of height, protuberant abdomen and height loss. Additionally, reflux eshophagitis and diminished pulmonary capacity may follow as a result of deformity. $^{6}$

The occurrence of an atraumatic vertebral fracture is generally sufficient to establish a diagnosis of osteoporosis. With increased understanding of bone fragility, it is now recognized that falls play a minor role in generating vertebral fractures. ${ }^{9}$ Postmenopausal women with one or more radiographically identified prevalent vertebral fractures have a highly increased risk of sustaining an incident vertebral fracture; the risk increases further with increasing number of prevalent fractures. ${ }^{10,11}$

\section{CONSEQUENCES OF OSTEOPOROTIC FRACTURES}

Osteoporotic fractures occur at many skeletal sites, although those of the hip, spine, wrist, and proximal humerus are the most common. Among Caucasians in the United States, the lifetime risk of fracture at age 50 years is at least $40 \%$ in women and $13 \%$ in men. ${ }^{12} \mathrm{Hip}$ fractures are the major cause of morbidity and mortality associated with osteoporosis. For instance, up to one quarter of hip fracture patients die within 1 year of their fracture. As many as half of all patients with a hip fracture will have long-term disability, and $25 \%$ will require long-term nursing home care. These patients may develop additional complications, including pressure ulcers, pneumonia, deep vein thrombosis and fatal pulmonary embolism, urinary tract complications, and depression. Although the rate of hip fracture among men is one third to one half that of women of similar age, the increased mortality associated with hip fracture is higher in men than women. ${ }^{13}$ Vertebral fractures are two to three times more prevalent than hip fractures, yet only about one third of vertebral deformities are diagnosed acutely. ${ }^{14}$ This may be because these fractures are sometimes associated with only minor symptoms or are asymptomatic and, therefore, may be overlooked by the physician. However, vertebral fractures can be extremely painful, debilitating, and frequently associated with loss of height, postural changes, kyphosis, decrease lung capacity, impairs normal physical functioning and reduced quality of life. Likewise patients feel loss of social role due to unable to participate in social activities and psychologically they can suffer with anxiety, depression, negative impact on an individual's self-esteem and self-image, and reduced health related quality of life. Patients with vertebral fractures often experience difficulties in performing simple activities such as standing, bending, walking, dressing, carrying, getting into and out of bed, cooking, washing, using the toilet, and reaching the floor. ${ }^{15-17}$ Like hip fractures, they are associated with increased mortality and morbidity. Patients with a non-spine fracture have approximately a twofold greater risk for future fractures than do individuals who have not had a fracture. Furthermore, up to half of patients with a prior vertebral fracture will experience additional vertebral fractures within 3 years, many within the first year. Compared with individuals with no history of vertebral fracture, a patient with a prior vertebral fracture has nearly a fivefold increased risk of future vertebral fractures and a two- to threefold increased risk of hip and other nonvertebral fractures. ${ }^{18}$ Altogether, patients with a history of any type of prior fragility fracture have a two to four fold increased risk of subsequent fractures compared with those without a previous fracture. 


\section{DIAGNOSIS}

\section{Laboratory Tests}

These should be started from routine investigations like, blood counts, liver function test, serum creatinine, urea, calcium, phosphorous, and alkaline phosphatase, to rule out other causes of osteoporosis.

\section{Radiography}

Decreased bone density can be detected only after $30 \%$ to $40 \%$ bone loss, but it is nonspecific. Vertebral deformities include loss of anterior height causing wedging or flattening and presence of cod-fish vertebra as a result of expansion of intervertebral spaces.

\section{Bone Mineral Density Measurement}

This is the most sensitive and accurate method to document osteoporosis. Bone densitometry can detect presence of decreased bone density, future fracture risks, and change in bone density over the time.

Varieties of techniques are available for measuring Bone mineral density (BMD).These include central techniques such as dual-energy x-ray densitometry (DXA) and quantitative computed tomography (QCT), as well as peripheral techniques such as radiographic absorptiometry (RA), quantitative ultrasound (QUS), and peripheral DXA (pDXA). Central techniques for measuring BMD have been advocated as more accurate predictor of fracture risk, but the most recent data indicate that less costly, more accessible peripheral BMD measurement are also effective predictors of fracture risk. ${ }^{19}$ However, only central techniques are appropriate for evaluating the response of BMD to treatment. Several studies have shown that the risk of vertebral fracture increases with decreasing BMD. ${ }^{20,21}$ When BMD values are adjusted for mean differences between the sexes, the fracture risk at a given BMD level is similar between men and women. This suggests that women may have increased risk of fracture compared to men because, in general, women's spinal BMD is lower than that of similarly-aged men. ${ }^{20}$

\section{Central Techniques}

\section{Dual-energy $x$-ray bone densitometry}

DXA can be used to measure density from hip or spine. This is the gold-standard technique for the measurement of bone mineral density due to its low radiation burden, high predictive value for fractures, and cost effectiveness.

\section{Quantitative computed tomography}

QCT is more expensive than DXA and involves higher radiation burden. QCT is the only technique that provides a threedimensional display of the data and can determine the true volu- metric BMD; all other techniques provide areal density. ${ }^{22} \mathrm{QCT}$ enables an integrated measurement of combined cortical and trabecular bone and also provides separate measurement for cortical and trabecular bone densities.

\section{Peripheral techniques}

Radiographic absorptiometry (RA) uses standard hand x-ray films together with a standardized aluminium wedge to enable measurement of phalangeal BMD. It is relatively accessible and inexpensive method.

Quantitative Ultrasound (QUS) is another relatively accessible and inexpensive method; it involves the measurement of the average speed of sound through bone and soft tissue. It is commonly performed at the heel or shin. Patellar ultrasound velocity is a significant predictor of incident vertebral deformity. ${ }^{23}$ Although there have been few studies specifically addressing the ability of QUS to predict vertebral fracture risk, its wide availability, low cost, and ability to be performed without exposing the patient to ionizing radiation make it an appealing choice for clinical osteoporosis screening.

Peripheral DXA (pDXA), an emerging technique for the assessment of BMD, pDXA can be conducted at the heel or forearm with similar ability to predict fracture risk as central DXA. It can be used as screening test to decide which patient need to be central DXA done.

\section{MANAGEMENT}

There are 3 goals of management: (1) to stop or reverse bone loss; (2) to increase or stabilize bone mass; and (3) to reduce fractures, pain, disability and mortality. ${ }^{24}$ However, while most of the focus is on treating osteoporosis management of osteoporosis involves prevention and treatment. The best treatment for osteoporosis is prevention. The risk of osteoporosis can be reduced by increasing peak bone mass or by decreasing the bone loss. ${ }^{25}$ Therefore, the most logical and cost-effective preventive strategies are to encourage young women to stop smoking and avoid excessive use of alcohol. They should also be counseled to exercise regularly and consume adequate amounts of calcium and vitamin D.

\section{Modalities}

Exercises - Weight bearing exercise should be encouraged. Exercise has efficacy in achieving peak bone mass, even in older patients. Activities such as aerobic dancing or running are better than swimming to increase bone mass. The exact type of exercise is less important than tailoring the prescription to the patient's individual needs. ${ }^{26}$ 
Life style modification: In addition to exercise, one should include discontinuing cigarettes smoking and avoiding excessive alcohol intake. Care to consume a nutritionally sound diet is important. The elderly are particularly at risk for malnutrition.

Calcium and Vitamin D: Ensuring adequate dietary calcium intake can be difficult if the patient does not consume a significant amount of dairy products, which are the primary source of calcium. Supplementation is advisable for women whose dietary intake is inadequate. Postmenopausal women are recommended to take $1500 \mathrm{mg}$ daily of calcium supplementation and 400 to 800 IU of vitamin D daily which is needed for adequate calcium absorption; those who are taking hormonal therapy are advised to take $1 \mathrm{~g}$ per day of Calcium. ${ }^{27}$ Calcium comes as a citrate or a carbonate formulation. Citrate is best absorbed on an empty stomach while the carbonate preparations should be taken with meals. Iron decreases absorption of calcium; thus, iron supplements should be taken at a different time of the day than the calcium.

Estrogen: Hormonal therapy (HT) has been used for a number of years because it has been demonstrated to increase BMD with subsequent prevention of fractures. The term hormone therapy has been suggested as a substitute for hormone replacement therapy because these medications do not replace the patient's normal hormones. Benefits of combined estrogen/progesterone exist in the short term to assist women with vasomotor symptoms related to the perimenopausal state..$^{28,29}$ Long-term estrogens alone appear to be beneficial in preventing osteoporosis. For years, HT was considered the primary preventive therapy for osteoporosis. Concerns over uterine cancer were minimized by the use of combined estrogen/progesterone therapies, and worries about the potential increased risk of breast cancer were minimized. ${ }^{30}$

One very large randomized prospective trial, the Women's Health Initiative (WHI), showed a 34\% reduction of hip fracture risk during hormone therapy (estrogen plus progestin) in normal postmenopausal women. ${ }^{31}$ This finding also was supported by several meta-analyses of other trials of hormone therapy, which confirmed a $33 \%$ reduction in vertebral fracture risk $^{32}$ and $13 \%$ to $27 \%$ reduction in nonvertebral fracture risk ${ }^{32,33}$ after hormone therapy.

Calcitonin: Calcitonin is a polypeptide hormone that inhibits bone reabsorption by its effect on osteoclasts. It is available as a nasal spray at a recommended dose of 200 IU per day, which corresponds to 1 squirt in each nostril every other day. It is also available in an injectable preparation given in 100 IU subcutaneously daily. Research has shown reduction of the risk of new vertebral fractures with the use of the nasal spray in postmenopausal women with osteoporosis. ${ }^{38}$
The largest clinical trial of osteoporotic women (1,108 patients) treated with nasal calcitonin showed small increases in BMD and a statistically significant $20 \%$ reduction in the risk of new vertebral fractures, with no effect on nonvertebral fractures. ${ }^{34}$ The drug is expensive and has got some side effects like flushing, nasal irritation, gastrointestinal symptoms and allergy. The drug is said to relieve pain after fracture, perhaps through circulating endorphins. Calcitonin is not as effective as biphosphonates, but is an alternative to estrogen in women who can not take estrogen.

Bisphosphonates: These are pyrophosphate analogues with antiresorptive properties. Several bisphosphonates are available for the treatment of postmenopausal osteoporosis, including alendronate, risedronate. These medications have been demonstrated to increase BMD and reduce risk for fractures. Fracture intervention trial done in Alendronate has shown to significantly reduce the impact on fractures of the spine and the hip in patients who had osteoporosis with baseline fractures of the spine. The reduction was about $50 \%$. Consequently, alendronate has been found an acceptable modality to treat osteoporotic patients because of this efficacy both in fractures of the spine and the hip. Bisphosphonates are associated with the risk for pill esophagitis, however, this may be modulated with the release of once-weekly regimens. It is important to continue calcium and vitamin D supplements while on these agents. Large randomized clinical trials of alendronate and risedronate have shown increase in BMD (3\% to $10 \%$ ) accompanied by statistically significant and substantial (up to $50 \%$ ) reductions in the risk of vertebral, hip, and other nonvertebral fractures in postmenopausal women with osteoporosis. ${ }^{35-38}$

Fluorides: Use remains controversial although it is one of the few medications which promote osteoblastic activity. It directly stimulates osteoblastic activity, and increases bone density but found bone to be more fragile.

Parathyroid Hormone: Parathyroid hormone (PTH) stimulates bone formation. Parathyroid hormone 1-34 is the only agent that is anabolic. Studies have shown that it can increase bone density in the lumbar spine. It needs to be administered via subcutaneous injection daily. PTH is indicated for patients who have severe osteoporosis with fractures, or for postmenopausal patients with osteoporosis who have failed to respond to other therapeutic alternatives. Daily subcutaneous administration of PTH (1-34) increases BMD and statistically significantly reduces the risk of new vertebral and non-vertebral fractures by $65 \%$ and $53 \%$, respectively, in osteoporotic women with an existing vertebral fracture. ${ }^{39}$ Several studies in animals suggest that PTH (1-34) also may enhance fracture healing itself, although this remains to be tested in humans. 


\section{REFERENCES}

1. National Institute of Health. Osteoporosis prevention, diagnosis, and therapy. Consensus Statement. Bethesda, MD: National Institutes of Health; 2000; 17:1-36.

2. Haczynski J, Jakimiuk A. Vertebral fractures: A hidden problems of osteoporosis. Med Sci monit 2001: 7: 1108-17.

3. Goddard D, Kleerekoper M. The epidemiology of osteoporosis. Post Grad. Medicine, 1988; 104: 54-70.

4. Mary L. Bouxsein, , John Kaufman, , Laura Tosi, ,Steven Cummings, Joseph Lane, and Olof Johnell. Recommendations for Optimal Care of the Fragility Fracture Patient to Reduce the Risk of Future Fracture. $J$ Am Acad Orthop Surg. 2004; 12(6): 385-395

5. Hurley DL, Khosla S: Update on primary osteoporosis. Mayo Clin Proc, 1977; 72: 943-949.

6. Off SM. Osteoporosis and Osteomalacia. In: Hazzard WR, Blass JP, Ettinger WH, Halter JB, Ouslander JG, Editors. Principle of Geriatric Medicine and Gerontology. New York: McGraw - Hill; 1999, 1057-1080.

7. Holland EFN, Stud JWW, Mansell JP et al. Changes in the collagen composition and cross-link in bone and skin of osteporotic post-menopausal women treated with percutaneous estradiol implants. Obstet Gynecol, 1994; 83: 180-3.

8. Goodman TA, Simon LS. Osteoporosis: Current issue in diagnosis and management. J Musculoskel Med. 1997; 14: 10-22.

9. Cooper C, Atkinson EJ, O'Fallon WM, Melton LJ III. Incidence of clinically diagnosed vertebral fractures: A population based study in Rochester, Minnesota, 1985-1989. J Bone Min Res. 1992; 7: 221-227.

10. Lindsay R, Silverman SL, Cooper $C$, et al. Risk of new vertebral fracture in the year following a fracture. JAMA. 2001; 285: 320-323.

11. Nevitt MC, Ross PD, Palermo L, Musliner T, Genant HK, Thompson $D E$. Association of prevalent fractures, bone density, and alendronate treatment with incident vertebral fractures: Effect of number and spinal location of fractures. Bone. 1999; 25: 613-619.

12. Melton LJ III, Chrischilles EA, Cooper C, Lane AW, Riggs BL: Perspective: How many women have osteoporosis? J Bone Miner Res 1992; 7 : 1005-1010.

13. Center JR, Nguyen TV, Schneider D, Sambrook PN, Eisman JA: Mortality after all major types of osteoporotic fracture in men and women: An observational study. Lancet 1999; 353: 878-882.

14. Black DM, Cummings SR, Karpf DB, et al: Randomised trial of effect of alendronate on risk of fracture in women with existing vertebral fractures: Fracture Intervention Trial Research Group. Lancet 1996; 348: 1535-1541.

15. Leidig G, Minne HW, Saur P,et al. A study of complains and their relation to vertebral destruction in patients with osteoporosis. Bone Miner. 1990; 8: $217-219$.

16. Cook DJ, Guyatt GH, Adachi JD, et al. Quality of life issue in women with vertebral fractures due to osteoporosis. Arth Rheum. 1993; 36: 750756.
17. Ryan PJ, Blake G, Herd R, Fogelman I. A clinical profile of back pain and disability in patients with spinal osteoporosis. Bone.1994; 15: 2730 .

18. Van Staa TP, Leufkens HG, Cooper C: Does a fracture at one site predict later fractures at other sites?ABritish cohort study. Osteoporos Int 2002; 13: 624-629.

19. Miller PD, Siris ES, Barret-Conner E, et al. Prediction of fracture risk in postmenopausal white women with peripheral bone densitometry: Evidence from the National Osteoporosis Risk Assessment. J Bone Miner Res. 2002; 17: 2222-2230.

20. The European Prospective Osteoporosis Study Group. The relationship between bone density and incident vertebral fracture in women and men. J Bone Miner Res. 2002; 17: 2214-2221.

21. Eddy D, Johnston C, Cummings SR, et al. Osteoporosis: Review of the evidence for prevention, diagnosis, and treatment and cost effective analysis. Osteoporosis Int. 1998; 8(suppl 4): S7-S80.

22. Kleerekoper M, Nelson DA. Which bone density measurement? J Bone Miner Res. 1997; 12: 712-714.

23. Heaney RP, Avioli LV, Chesnut CH, III, Lappe J, Recker RR, Brandenburger GH. Ultrasound velocity through bone predicts incident vertebral deformity. J Bone Miner Res. 1995; 10: 341-345.

24. Lambing CL. Osteoporosis 2003. Program and abstracts of the American Academy of Family Physicians 2003 Annual Scientific Assembly; October 1-5, 2003; New Orleans, Louisiana. Abstract 181.

25. Riggs BL, Melto LJ. The prevention and treatment of osteoporosis. N Eng J Med 1992; 327: 622-626.

26. Varga M. Osteoporosis: Strategies for prevention and treatment. J Musculoskel Med 1995; 12: 19-30.

27. Lambing CL. Osteoporosis 2003. Program and abstracts of the American Academy of Family Physicians 2003 Annual Scientific Assembly; October 1-5, 2003; New Orleans, Louisiana. Abstract 181.

28. Apgar BS. HRT dilemma. Program and abstracts of the American Academy of Family Physicians 2003 Annual Scientific Assembly; October 15, 2003; New Orleans, Louisiana. Abstract 12.

29. Hays J, Ockene JK, Brunner RL, et al, for the Women's Health Initiative Investigators. Effects of estrogen plus progestin on health-related quality of life. N Engl J Med. 2003; 348: 1839-1854.

30. Langer RD. Postmenopausal hormone therapy: the state-of-the-art 2003. Program and abstracts of the American Academy of Family Physicians 2003 Annual Scientific Assembly; October 1-5, 2003; New Orleans, Louisiana. Abstract, 567.

31. Rossouw JE, Anderson GL, Prentice RL, et al: Risks and benefits of estrogen plus progestin in healthy postmenopausal women: Principal results from theWomen's Health Initiative randomized controlled trial. JAMA 2002; 288: 321-333.

32. Wells G, Tugwell P, Shea B, et al: Metaanalyses of therapies for postmenopausal osteoporosis: V. Meta-analysis of the efficacy of hormone replacement therapy in treating and preventing osteoporosis in postmenopausal women. Endocr Rev, 2002; 23: 529-539. 
33. Torgerson DJ, Bell-Syer SE: Hormone replacement therapy and prevention of nonvertebral fractures:Ameta-analysis of randomized trials. JAMA 2001; 285: 2891-2897.

34. Chestnut $\mathrm{CH}$, Silverman $\mathrm{S}$, Andriano K, et al. A randomized trial of nasal spray salmon calcitonin in post-menopausal women with established osteoporosis: the prevention of recurrence of osteoporotic fractures study. PROOF Study Group. Am J Med. 2000; 109:267-276.

35. Black DM, Cummings SR, Karpf DB, et al: Randomised trial of effect of alendronate on risk of fracture in women with existing vertebral fractures: Fracture Intervention Trial Research Group. Lancet 1996; 348: 1535-1541.

36. Cranney A, Guyatt G, Griffith L, Wells G, Tugwell P, Rosen C: Metaanalyses of therapies for postmenopausal osteoporosis: IX.Summaryof meta-analyses of therapies for postmenopausal osteoporosis.Endocr Rev 2002; 23: 570-578.

37. Harris ST, Watts NB, Genant HK, et al: Effects of risedronate treatment on vertebral and nonvertebral fractures in women with postmenopausal osteoporosis: A randomized controlled trial. Vertebral Efficacy With Risedronate Therapy (VERT) Study Group. JAMA, 1999; 282: 13441352.

38. McClung MR, Geusens P, Miller PD, et al: Effect of risedronate on the risk of hip fracture in elderly women: Hip Intervention Program Study Group. N Engl J Med 2001; 344: 333-3.

39. Neer RM, Arnaud CD, Zanchetta JR, et al: Effect of parathyroid hormone (1-34) on fractures and bone mineral density in postmenopausal women with osteoporosis. N Engl J Med 2001; 344: 1434-1441. 